\title{
Comparative In Vitro and In Vivo Quantifications of Pathologic Tau Deposits and Their Association with Neurodegeneration in Tauopathy Mouse Models
}

\author{
Ruiqing $\mathrm{Ni}^{1-3}$, Bin $\mathrm{Ji}^{1}$, Maiko Ono ${ }^{1}$, Naruhiko Sahara ${ }^{1}$, Ming-Rong Zhang ${ }^{1}$, Ichio Aoki ${ }^{1}$, Agneta Nordberg ${ }^{2,3}$, \\ Tetsuya Suhara ${ }^{1}$, and Makoto Higuchi ${ }^{1}$ \\ ${ }^{I}$ National Institute of Radiological Sciences, National Institutes for Quantum and Radiological Science and Technology, Chiba, Chiba \\ 263-8555, Japan; ${ }^{2}$ Department of Neurobiology, Care Sciences and Society, Division of Clinical Geriatrics, Karolinska Institute, \\ Stockholm, Sweden; and ${ }^{3}$ Theme Aging, Karolinska University Hospital, Stockholm, Sweden
}

Fibrillary tau aggregates in Alzheimer disease and allied neurodegenerative disorders have been visualized in vivo by PET, whereas mechanistic links between PET-detectable tau deposits and neurotoxicity remain elusive. Here, we took advantage of transgenic mouse models of tauopathies to evaluate associations between PET and postmortem measures of tau probe binding and their relation to neuronal loss. Methods: PET with a tau probe, ${ }^{11} \mathrm{C}-\mathrm{PBB} 3$ (2-((1E,3E)-4-(6-( ${ }^{11} \mathrm{C}-$ methylamino)pyridine-3-yl)buta-1,3-dienyl) benzo[d]thiazol-6-ol), and volumetric MRI were performed for transgenic $\mathrm{rTg} 4510$ mice and nontransgenic mice. Binding of ${ }^{11} \mathrm{C}-\mathrm{PBB} 3$ and its blockade by another tau binding compound, AV-1451 (-(6fluoropyridine-3-yl)-5H-pyrido[4,3-b]indole), in homogenized brains of tauopathy patients and rTg4510 and PS19 mice were quantified, and ${ }^{11} \mathrm{C}$-PBB3-positive and phosphorylated tau lesions in sectioned brains of these mice were assessed. Results: In vivo ${ }^{11} \mathrm{C}$-PBB3 binding to the $\mathrm{rTg} 4510$ neocortex/hippocampus was increased relative to controls and correlated with local atrophy. In vitro ${ }^{11} \mathrm{C}$-PBB3 binding in the neocortex/hippocampus also correlated well with in vivo radioligand binding and regional atrophy in the same individual rTg4510 mice. By contrast, in vitro ${ }^{11} \mathrm{C}$-PBB3 binding was elevated in the brain stem but not hippocampus of PS19 mice, despite a pronounced loss of neurons in the hippocampus rather than brain stem. Finally, ${ }^{11} \mathrm{C}-\mathrm{PBB} 3$ and AV-1451 showed similar binding properties between mouse models and tauopathy patients. Conclusion: The present findings support the distinct utilities of ${ }^{11} \mathrm{C}$-PBB3 PET and MRI in rTg4510 and PS19 mice for quantitatively pursuing mechanisms connecting PET-detectable and PET-undetectable tau aggregations to neuronal death, which recapitulate 2 different modes of tau-provoked neurotoxicity.

Key Words: tauopathy; transgenic mouse model; small-animal PET; volumetric MRI; radioligand binding

J Nucl Med 2018; 59:960-966

DOI: 10.2967/jnumed.117.201632
D eposition of pathologic tau fibrils is characteristic of Alzheimer disease $(\mathrm{AD})$ and related non- $\mathrm{AD}$ neurodegenerative disorders as exemplified by frontotemporal lobar degeneration (1). Normally functioning tau proteins are constituents of axonal cytoskeletons in neurons, and the self-assembly of tau molecules in a diseased condition gives rise to disruption of the axonal framework along with neurotoxicity induced by misfolded tau species $(1,2)$. In line with this mechanistic view, tau abnormalities are known to be tightly associated with neurodegeneration and the emergence of clinical symptoms $(3,4)$, providing a rationale for antitau treatments to modify the disease process (5). For the purpose of establishing etiologic, diagnostic, and therapeutic assessments of tau pathologies in living subjects, there have been growing demands for the visualization of tau fibrils in a noninvasive manner.

In vivo PET of tau lesions has recently been enabled by the development of small-molecule imaging agents selectively binding to the $\beta$-pleated sheet structure in tau filaments (6). Several tau PET probes, including ${ }^{11} \mathrm{C}-\mathrm{PBB} 3\left(2-\left((1 \mathrm{E}, 3 \mathrm{E})-4-\left(6-\left({ }^{11} \mathrm{C}-\right.\right.\right.\right.$ methylamino $)$ pyridine-3-yl)buta-1,3-dienyl)benzo[d]thiazol-6-ol) (7), ${ }^{18} \mathrm{~F}-\mathrm{THK} 5117$ (8), ${ }^{18} \mathrm{~F}-\mathrm{THK} 5351$ (9), and ${ }^{18} \mathrm{~F}-\mathrm{AV}-1451$ (-(6-fluoropyridine-3-yl)-5Hpyrido[4,3-b]indole) (also known as ${ }^{18} \mathrm{~F}-\mathrm{T} 807$ or ${ }^{18} \mathrm{~F}$-flortaucepir) (1012), have been applied to human subjects across the $\mathrm{AD}$ spectrum and have offered neuroimaging-based staging of tau pathologies. In these individuals, the distribution of PET-detectable tau deposits was reported to agree with the topography of brain atrophy and manifestation of focal symptoms $(7,9,12)$, implicating the tau aggregation in the local neuronal deteriorations. In the meantime, it still remains elusive whether tau deposits in frontotemporal lobar degeneration are sensitively detectable by these PET probes to examine links between the localization of tau pathologies and clinical phenotypes (13-17). It is noteworthy that tau proteins comprise 6 isoforms classified into 4-repeat and 3-repeat species, and the composition of tau isoforms differs among diverse tauopathies $(1,2)$. Indeed, AD-type tau fibrils are constituted by all 6 isoforms, in contrast to the exclusive assembly of 4-repeat tau isoforms into fibrils in a subgroup of frontotemporal lobar degeneration, such as progressive supranuclear palsy (PSP) and corticobasal degeneration $(1,2)$. These distinct tau fibril species are ultra-structurally identified as paired helical filaments in $\mathrm{AD}$ and straight filaments in 4-repeat tauopathies $(18,19)$, and such conformational variations may affect the reactivity of tau fibrils with PET probes (13). In fact, binding of ${ }^{11} \mathrm{C}-\mathrm{PBB} 3$ in the 
PSP motor cortex is not blocked by AV-1451, in contrast to a partial blockade of ${ }^{11} \mathrm{C}-\mathrm{PBB} 3$ binding by $\mathrm{AV}-1451$ in the $\mathrm{AD}$ temporal cortex. The binding characteristics of these ligands can accordingly be used for discrimination between AD-like and PSP-like tau aggregates in human and mouse brain tissues.

In the nonclinical development of ${ }^{11} \mathrm{C}-\mathrm{PBB} 3$ (7), we used mice transgenic for a human 4-repeat tau isoform with the P301L and P301S mutations, termed the $\mathrm{rg} 4510(\mathrm{Tg}=$ transgenic) $(20)$ and PS19 (21) lines, respectively. In vivo PET imaging and ex vivo autoradiographic imaging, and in vivo and ex vivo fluorescence imaging, of these animals provided significant information on the kinetics and detectability of tau deposits in the living brain by a candidate imaging agent (7). However, it still remains unclear whether preclinical investigation using rTg4510 and PS19 combined with ${ }^{11} \mathrm{C}-\mathrm{PBB} 3$ PET could provide useful information on imaging-pathologic relationships as in a clinical PET study or potentially contribute to quantitatively understanding the mechanisms connecting PET-detectable and PET-undetectable tau aggregations to neuronal death. In addition, the radioligand binding in small-animal PET assays might be underestimated in small anatomic structures because of partial-volume effects and might be overestimated in surface brain areas because of radioactivity spillin from extracranial tissues. PET measures should accordingly be validated with reference to postmortem binding estimates in the same individuals.

To address these issues, we conducted ${ }^{11} \mathrm{C}-\mathrm{PBB} 3$ PET and volumetric MRI on rTg4510 mice, followed by postmortem assessments of the brains from the same animals, and assessed whether in vivo PET signals reflect the amount of specific ${ }^{11} \mathrm{C}$-PBB3 binding and phosphorylated tau deposits. Associations of these binding estimates with cerebral atrophy determined by MRI were also analyzed to demonstrate the ability of ${ }^{11} \mathrm{C}$-PBB3 PET to quantify tau species tightly linked to the neurotoxicity. Similarly, in vitro binding assays were applied to brain tissues collected from PS19 mice to clarify whether toxicity-related tau assemblies can be captured by ${ }^{11} \mathrm{C}-\mathrm{PBB} 3$ across different tauopathy models. Finally, in vitro binding of ${ }^{11} \mathrm{C}-\mathrm{PBB} 3$ versus $\mathrm{AV}-1451$ in $\mathrm{rTg} 4510$ and PS19 mouse samples was compared with those of AD and PSP tissues for examining the translatability of the probe binding data in mouse models to AD and 4-repeat tauopathies. Assessments of the competition between ${ }^{11} \mathrm{C}-\mathrm{PBB} 3$ and AV-1451 binding in various brain tissues would also provide information on the presence of common off-target binding sites for these 2 compounds.

\section{MATERIALS AND METHODS}

\section{Ethics Statement}

The mice studied here were maintained and handled in accordance with the National Research Council's Guide for the Care and Use of Laboratory Animals and our institutional guidelines. Protocols for the present animal experiments were approved by the Animal Ethics Committees of the National Institute of Radiologic Sciences, National Institutes for Quantum and Radiologic Science and Technology. The research using autopsied human samples was approved by the Ethics Committee of National Institute of Radiologic Sciences.

\section{Reagents and Antibody}

PBB3 and a desmethyl precursor for radiosynthesis of ${ }^{11} \mathrm{C}-\mathrm{PBB} 3$ were obtained from Nard Institute. AV-1451 was in-house-synthesized according to a previous report (US 2012/0302755 A1). BTA-1 (2-(4'-methylaminophenyl)benzothiazole), clorgyline, and selegiline (also known as deprenyl) were purchased from Sigma-Aldrich. AT8, a mouse monoclonal antibody against human tau phosphorylated at Ser202 and Thr205, was purchased from Endogen/Thermo Fisher Scientific.

\section{Animal Models}

Two mouse models, PS19 and $\mathrm{rTg} 4510$, were used in the present study. PS19 transgenic mice heterozygous for human T34 (4-repeat tau isoform with a single N-terminal insert), with P301S mutation driven by the mouse prion protein promoter (21), were provided by the Perelman School of Medicine, University of Pennsylvania, and were bred and kept on a C57BL/6 background. rTg4510 mice, tau responder mice, and tetracycline-controlled transactivator mice were obtained from the University of Florida. A parental mutant tau responder line on an FVB/N strain (Clea Inc.) and a tetracyclinecontrolled transactivator activator line in a $129+$ ter/SV strain (Clea Inc.) were generated and maintained. To generate a tau responder line expressing human $\mathrm{T} 43$ (4-repeat tau isoform without N-terminal inserts) with P301L mutation, complementary DNA was placed downstream to a tetracycline-operon-responder construct. The tetracyclinecontrolled transactivator system was inserted downstream to the $\mathrm{Ca}^{2+}$-calmodulin kinase II $\alpha$ promoter. Hemizygous mice from each parental line were cross-bred to produce $\mathrm{rTg} 4510$ mice possessing both responding and tetracycline-controlled transactivator constructs and nontransgenic littermates (20). A workflow of in vivo PET and MRI and in vitro binding assays with $\mathrm{rTg} 4510$ and nontransgenic mice is shown in Supplemental Figure 1 (supplemental materials are available at http://jnm.snmjournals.org).

\section{Radiosynthesis and Small-Animal PET Imaging}

Radiosynthesis of ${ }^{11} \mathrm{C}$-PBB3 was performed as described previously (7), and the resulting radiochemical purity was more than $90 \%$. The molar activity of ${ }^{11} \mathrm{C}-\mathrm{PBB} 3$ was $78-93.1 \mathrm{GBq} / \mu \mathrm{mol}$ at the end of synthesis. PET was performed on a microPET Focus 220 animal scanner (Siemens Medical Solutions) as described previously (22). At 7-10 mo old, $r T g 4510$ mice $(n=5$; body weight, $23.6 \pm 0.8 \mathrm{~g})$ and age-matched nontransgenic mice ( $n=7$; body weight, $29.1 \pm 1.5 \mathrm{~g})$ were anesthetized with $1.5 \%(\mathrm{v} / \mathrm{v})$ isoflurane. Emission scans were acquired for $60 \mathrm{~min}$ in 3-dimensional list mode with an energy window of $350-750 \mathrm{keV}$, immediately after intravenous injection of ${ }^{11} \mathrm{C}-\mathrm{PBB} 3$ (18.2-23.4 MBq/mouse; $20.76 \pm 2.21 \mathrm{MBq}$ for nontransgenic mice and $21.24 \pm 1.24 \mathrm{MBq}$ for transgenic mice). Images were reconstructed by either a maximum-a-posteriori method to generate single-frame average images for nonquantitative displays or filtered backprojection using a 0.5-mm Hanning filter to generate dynamic images for quantitative assays. All transgenic and 4 nontransgenic mice were scanned by T2weighted MRI within $1 \mathrm{wk}$ of the PET scans. Volumes of interest (VOIs) were placed on multiple anatomic structures, including the neocortex (coronal sections from $1.3 \mathrm{~mm}$ anterior to $2.7 \mathrm{~mm}$ posterior to the bregma), whole hippocampus, and cerebellum (6.4-7.0 mm posterior to the bregma) using PMOD image analysis software (PMOD Technologies LLC) with reference to individual T2-weighted MR images for all transgenic and 4 nontransgenic mice or a brain template generated as described previously (7) for the other 3 nontransgenic mice (Fig. 1A and Supplemental Fig. 2). The PET VOIs did not contain areas near the olfactory bulbs to circumvent radioactivity spillover from the Harderian glands. The neocortex more than $2.7 \mathrm{~mm}$ posterior to the bregma was excluded from the PET VOIs, since profound atrophy of this region in an aged rTg4510 mouse caused a partial-volume effect on PET data, impeding an accurate quantification of the radioligand retention. Tracer uptake in each VOI was estimated as percentage injected dose per tissue volume, which was uncorrected for body weights of animals, since changes in body weights during aging may not necessarily be proportional to changes in brain weights. Nondisplaceable binding potential for ${ }^{11} \mathrm{C}-\mathrm{PBB} 3$ binding in these VOIs, which is not influenced by either body or brain weights, 


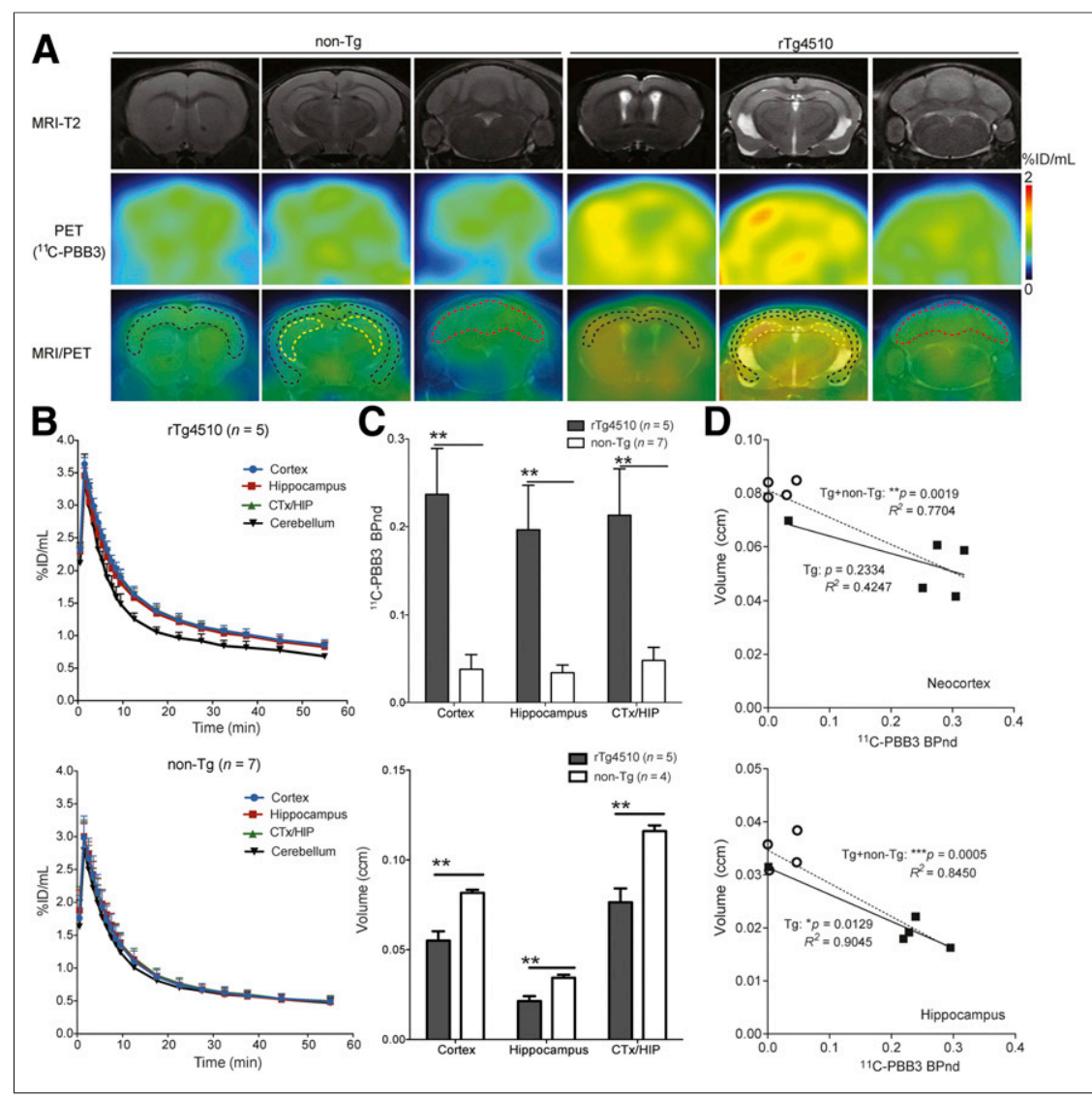

FIGURE 1. Correlations of PBB3-positive tau deposits with neocortical/hippocampal atrophy in rTg4510 mouse brain. (A) Representative T2-weighted MR, PET, and PET/MR images of coronal brain sections of 9-mo-old nontransgenic and rTg4510 mice showing neocortical, hippocampal, and cerebellar VOIs (black, yellow, and red outlines, respectively). PET images were generated from averaged dynamic data at $30-60 \mathrm{~min}$ after injection of ${ }^{11} \mathrm{C}$-PBB3. (B) Time-activity curves (percentage injected dose [\%ID]) for ${ }^{11} \mathrm{C}$-PBB3 in different brain regions of $\mathrm{rTg} 4510$ and agematched nontransgenic mice at $7-10$ mo old. (C) ${ }^{11} \mathrm{C}$-PBB3 binding potential in each VOI calculated by simplified reference tissue model with cerebellum as reference tissue using data from $B$, and brain volume measured using structural MRI data, including calculation of nondisplaceable binding potential for neocortex and hippocampus (CTX/HIP). ${ }^{*}$ Significant increase in ${ }^{11} \mathrm{C}-\mathrm{PBB} 3$ nondisplaceable binding potential, and significant decrease in neocortical and hippocampal volume, for rTg4510 mice compared with nontransgenic mice $(P<0.01)$. (D) Correlation between ${ }^{11} \mathrm{C}$-PBB3 nondisplaceable binding potential and volume of neocortex and hippocampus in transgenic ( $(\square)$ and nontransgenic $(O)$ mice. ${ }^{\star} P<0.05,{ }^{\star \star} P<0.01$, and ${ }^{\star \star \star} P<0.001$ for correlations in transgenic plus nontransgenic group ( $\mathrm{Tg}+$ non $\mathrm{Tg}$, dotted lines) and transgenic group only ( $\mathrm{Tg}$, solid lines).

was quantified with PMOD based on a simplified reference tissue model using the cerebellum as a reference region lacking tau deposits.

\section{MRI of Mouse Brains}

The $\mathrm{rTg} 4510$ mice were anesthetized with $1.5 \%(\mathrm{v} / \mathrm{v})$ isoflurane. T2-weighted 2-dimensional multislice spin-echo (rapid acquisition with relaxation enhancement [RARE]) was applied to the mouse heads using the 7.0-T MRI system (AVANCE-III; Bruker BioSpin) with a volume coil for transmission (Bruker BioSpin) and a quadrature surface coil for reception (Rapid Biomedical). The repetition time was 4,200 ms, effective echo time was $36 \mathrm{~ms}$, field of view was $25.6 \times$ $14.5 \mathrm{~mm}$, slice thickness was $0.5 \mathrm{~mm}$, number of slices was 28 (gapless), matrix was $256 \times 256$, RARE factor was 8 , number of acquisitions was 8 , and nominal in-plane resolution was $100 \times 57 \mu \mathrm{m}$. VOIs across all slices of the hippocampus and cortex were manually drawn with reference to the mouse brain atlas of Paxinos and Franklin using PMOD software.
In Vitro ${ }^{11} \mathrm{C}-\mathrm{PBB} 3$ Binding Assays

All $\mathrm{rTg} 4510$ and nontransgenic mice were killed by cervical dislocation after the scans. Brains were sampled from 3 PS19 and 3 nontransgenic mice at 13 mo old. The mouse brains were removed and divided into right and left hemispheres. The right hemispheres were kept at $-80^{\circ} \mathrm{C}$ for postmortem analyses, and the left hemispheres were fixed with $4 \%$ paraformaldehyde in phosphate buffer overnight, followed by $30 \%$ sucrose in phosphate buffer. Brain tissue from patients with $A D$ and PSP were homogenized using MicroSmash (MS-100R; TOMY Digital Biology) in $50 \mathrm{mM}$ Tris-HCl buffer ( $\mathrm{pH}$ 7.4) containing a protease/phosphatase inhibitor cocktail (cOmplete; Sigma-Aldrich). Concentrations of proteins in the brain homogenates were measured by bicinchoninic acid assays (Thermo Fisher Scientific). Aliquots of the homogenates were stored at $-80^{\circ} \mathrm{C}$ until the experiment.

Radioligand binding was measured by incubating $5 \mathrm{nM}{ }^{11} \mathrm{C}-\mathrm{PBB} 3$ with brain tissue homogenates from 5 rTg4510 and 7 nontransgenic mice that had undergone a PET scan, as well as from 3 PS19 and 3 nontransgenic mice. Nonspecific binding was determined in the presence of $5 \times 10^{-7} \mathrm{M}$ unlabeled PBB3.

Homologous and heterologous competitive binding assays were performed: $100-\mu \mathrm{L}$ aliquots of the tissue homogenates containing $100 \mu \mathrm{g}$ of AD brain tissue or $300 \mu \mathrm{g}$ of mouse brain tissue were reacted with $900 \mu \mathrm{L}$ of $50 \mathrm{mM}$ Tris- $\mathrm{HCl}$ buffer ( $\mathrm{pH}$ 7.4) containing $10 \%$ ethanol and $5 \mathrm{nM}{ }^{11} \mathrm{C}-\mathrm{PBB} 3$ in the presence of various unlabeled compounds, including PBB3, BTA-1, AV-1451, clorgyline, and selegiline at concentrations ranging from $10^{-11}$ to $10^{-6} \mathrm{M}$, for $30 \mathrm{~min}$ at room temperature in a dimly lit room to avoid photoisomerization of the compound. Nonspecific radioligand binding was determined in the presence of $5 \times 10^{-7}$ M PBB3. Samples were run in duplicates, and the specific radioligand binding was expressed as $\mathrm{pmol} / \mathrm{g}$ of tissue.

The inhibitory constant $\left(\mathrm{K}_{\mathrm{i}}\right)$, which is equivalent to the dissociation constant $\left(\mathrm{K}_{\mathrm{D}}\right)$ in the homologous blocking assay, and the percentage of displacement were determined using nonlinear regression 1-site and 2-site binding models derived from the Cheng-Prusoff equation in Prism, version 5.0 (GraphPad Software), followed by an $F$ test for model selection.

\section{Immunohistochemical and Histochemical Analyses}

Ten-micrometer-thick frozen sections from left hemispheres were generated in a cryostat (HM560; Carl Zeiss) and used for immunostaining with an antibody against phosphorylated tau (AT8; 1:250 dilution) by following a standard immunohistochemical procedure described in our previous publications (23). Fluorescence microscopic visualization of tau fibrils with PBB3 and FSB was performed as described previously (7). All stained samples were examined by an all-in-one fluorescence microscope (BZ-9000; Keyence) capable of tiling photomicrographs and merging them into a high-resolution image with a large field of view. 


\section{Statistics}

Statistical analyses for comparison of mouse genotype groups were performed by unpaired $t$ testing using Prism 5.0. The difference between groups was considered significant at a $P$ value of less than 0.05 . All error bars in the figures are expressed as SEM.

\section{RESULTS}

\section{Correlation Between Increased In Vivo ${ }^{11} \mathrm{C}-\mathrm{PBB} 3$ Binding and Brain Atrophy in rTg4510 Mice}

Images of ${ }^{11} \mathrm{C}-\mathrm{PBB} 3$ PET and structural MRI demonstrated overtly increased radioactivity retention and decreased volume in either neocortex or hippocampus of rTg4510 mice compared with age-matched nontransgenic mice (Fig. 1A). Time-activity curves also indicated increased ${ }^{11} \mathrm{C}$-PBB3 retention in the neocortex/hippocampus relative to the cerebellum of $\mathrm{rTg} 451$ mice, whereas no overt regional difference in radioligand retention was observed in nontransgenic mice (Fig. 1B). Nondisplaceable binding potential for ${ }^{11} \mathrm{C}$-PBB3 was significantly increased by 3 to 5-fold in the neocortex and hippocampus of rTg4510 compared with nontransgenic mice (Fig. 1C). This change was concurrent with marked reductions of the neocortical (approximately 33\%) and hippocampal (approximately 38\%) volumes in rTg4510 mice relative to nontransgenic mice (Fig. 1C). Indeed, intimate correlations

between ${ }^{11} \mathrm{C}$-PBB3 nondisplaceable binding potential and the volume of either neocortex or hippocampus were found in an analysis of combined transgenic and nontransgenic mice. This approach was made by conceiving nontransgenic mice to be animals at stage 0 of the tau pathology. In an assessment of the transgenics only, nondisplaceable binding potential values were still significantly correlated with local volumes in the hippocampus and showed a tendency to correlate with each other in the neocortex, despite a small sample size (Fig. 1D).

\section{Postmortem Assays of Tau Pathologies in rTg4510 and PS19 Mice}

Abundant neuronal inclusions fluorescently stained with PBB3 and immunolabeled with AT8 were observed in the neocortex and hippocampus but not in the cerebellum (data not shown) of 7- to 10mo-old rTg4510 mice. Similarly, accumulation of PBB3- and AT8positive tau deposits was detected in the brain stem of 13-mo-old PS19 mice. There was a lack of intense fluorescence labeling with PBB3 in the hippocampus of these PS19 mice despite abundant immunostaining with AT8 (Fig. 2A). In vitro ${ }^{11} \mathrm{C}-\mathrm{PBB} 3$ binding was also increased in the neocortex/hippocampus of 7- to 10-mo-old rTg4510 mice and mixed brain stem/spinal cord tissues of 13-moold PS19 mice compared with corresponding nontransgenic mice, whereas ${ }^{11} \mathrm{C}$-PBB3 binding in the mixed neocortex/hippocampus tissues of these PS19 mice was unchanged relative to age-matched nontransgenic mice

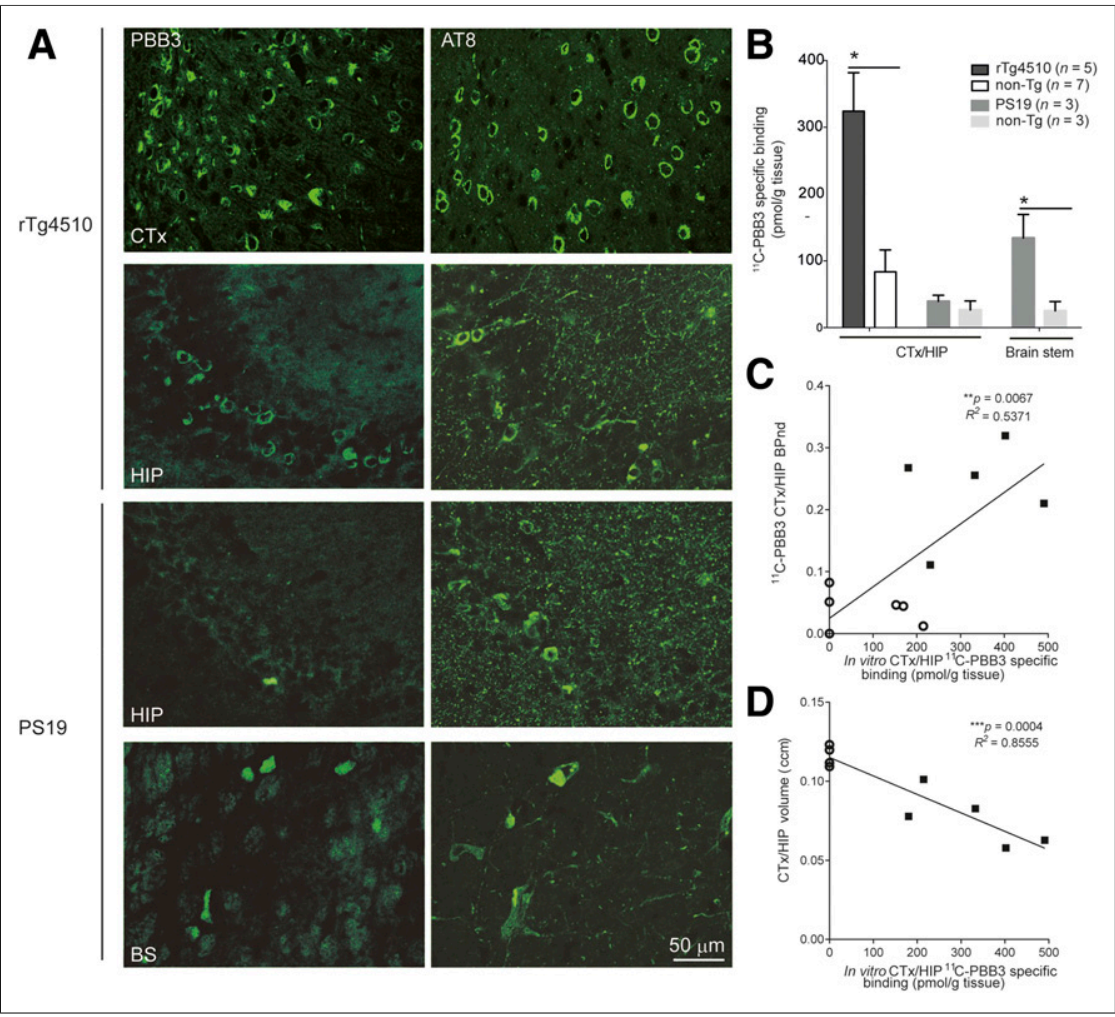

FIGURE 2. Comparisons of in vitro ${ }^{11} \mathrm{C}$-PBB3 binding and histochemical and immunohistochemical findings in brains of $\mathrm{rTg} 4510$ and PS19 mice. (A) Representative images of PBB3positive tau deposits and phosphorylated tau (AT8) in cortex (CTx), hippocampus (HIP), and brain stem (BS) of 9-mo-old rTg4510 mice and 13-mo-old PS19 mice. (B) Increased specific binding of ${ }^{11} \mathrm{C}-\mathrm{PBB} 3(5 \mathrm{nM})$ in CTX/HIP of $\mathrm{rTg} 4510$ mice and in brain stem of PS19 mice compared with nontransgenic mice $\left({ }^{*} P<0.05\right)$. No significant difference in ${ }^{11} \mathrm{C}$-PBB3 binding in CTX/HIP was observed between PS19 and nontransgenic mice. (C and D) Significant correlation of in vitro ${ }^{11} \mathrm{C}$ PBB3 binding with in vivo nondisplaceable binding potential $(C)$ and regional volume (D) in CTX/ HIP of $r \operatorname{Tg} 4510(\boldsymbol{\square})$ and nontransgenic $(\odot)$ mice.
(Fig. 2B). In vitro ${ }^{11} \mathrm{C}-\mathrm{PBB} 3$ binding showed a good correlation with both in vivo ${ }^{11} \mathrm{C}$ PBB3 binding and volume reduction in the neocortex/hippocampus of $\mathrm{rTg} 4510$ and nontransgenic mice (Figs. 2C and 2D).

\section{Characteristics of Tau Fibrils in Mouse Models Versus AD and PSP Based on Binding of PBB3 and AV-1451}

We assumed that conformational features of tau fibrils in the brain could be probed by their reactivity with different ligands. Following this rationale, ${ }^{11} \mathrm{C}-\mathrm{PBB} 3$ binding in homogenized frontal cortical tissues of an AD patient with abundant amyloid plaques and neurofibrillary tangles in the frontal cortex (Supplemental Fig. 3) was measured under a homologous blockade by nonlabeled PBB3 and a heterologous blockade by AV-1451 or BTA-1. The homologous blocking of ${ }^{11} \mathrm{C}-\mathrm{PBB} 3$ binding by nonlabeled $\mathrm{PBB} 3$ indicated the presence of high- and low-affinity binding components for this ligand, with $\mathrm{K}_{\mathrm{i}}\left(=\mathrm{K}_{\mathrm{D}}\right)$ values of 3.9 and $246.6 \mathrm{nM}$, respectively (Fig. 3A). The heterologous blockade of ${ }^{11} \mathrm{C}-\mathrm{PBB} 3$ binding by BTA-1, which is more selective for $A \beta$ versus tau filaments, was well described by a 1 -site model, with a $\mathrm{K}_{\mathrm{i}}$ value of $79.0 \mathrm{nM}$ (Fig. 3A). According to this inhibition curve, BTA-1 at a concentration below $50 \mathrm{nM}$ in the AD brain, which could reflect its high-affinity binding to $A \beta$ aggregates, minimally blocked the specific 
${ }^{11} \mathrm{C}-\mathrm{PBB} 3$ binding. AV-1451 displaced $30 \%-40 \%$ of ${ }^{11} \mathrm{C}-\mathrm{PBB} 3$ binding at maximum in the AD tissue (Fig. 3A). This blockade was described by a 1-site model with a $\mathrm{K}_{\mathrm{i}}$ value of $89.6 \mathrm{nM}$, which was approximately 50-fold weaker than the homologous blockade by nonlabeled PBB3. In the PSP basal ganglia tissue, a 1-site model indicated high-affinity binding of ${ }^{11} \mathrm{C}-\mathrm{PBB} 3$ to tau deposits, with a $K_{i}\left(=K_{D}\right)$ value of $2 \mathrm{nM}$, and this was barely blocked by $\mathrm{AV}-1451$ even at a high concentration (Fig. 3B).

Likewise, ${ }^{11} \mathrm{C}-\mathrm{PBB} 3$ exhibited high-affinity binding to tau deposits in the neocortex/hippocampus of rTg4510 mice (Fig. 3C) and the brain stem/spinal cord of PS19 mice (Fig. 3D), with $\mathrm{K}_{\mathrm{i}}$ $\left(=\mathrm{K}_{\mathrm{D}}\right.$ ) values of 1.8 and $1.3 \mathrm{nM}$, respectively, and this radioligand

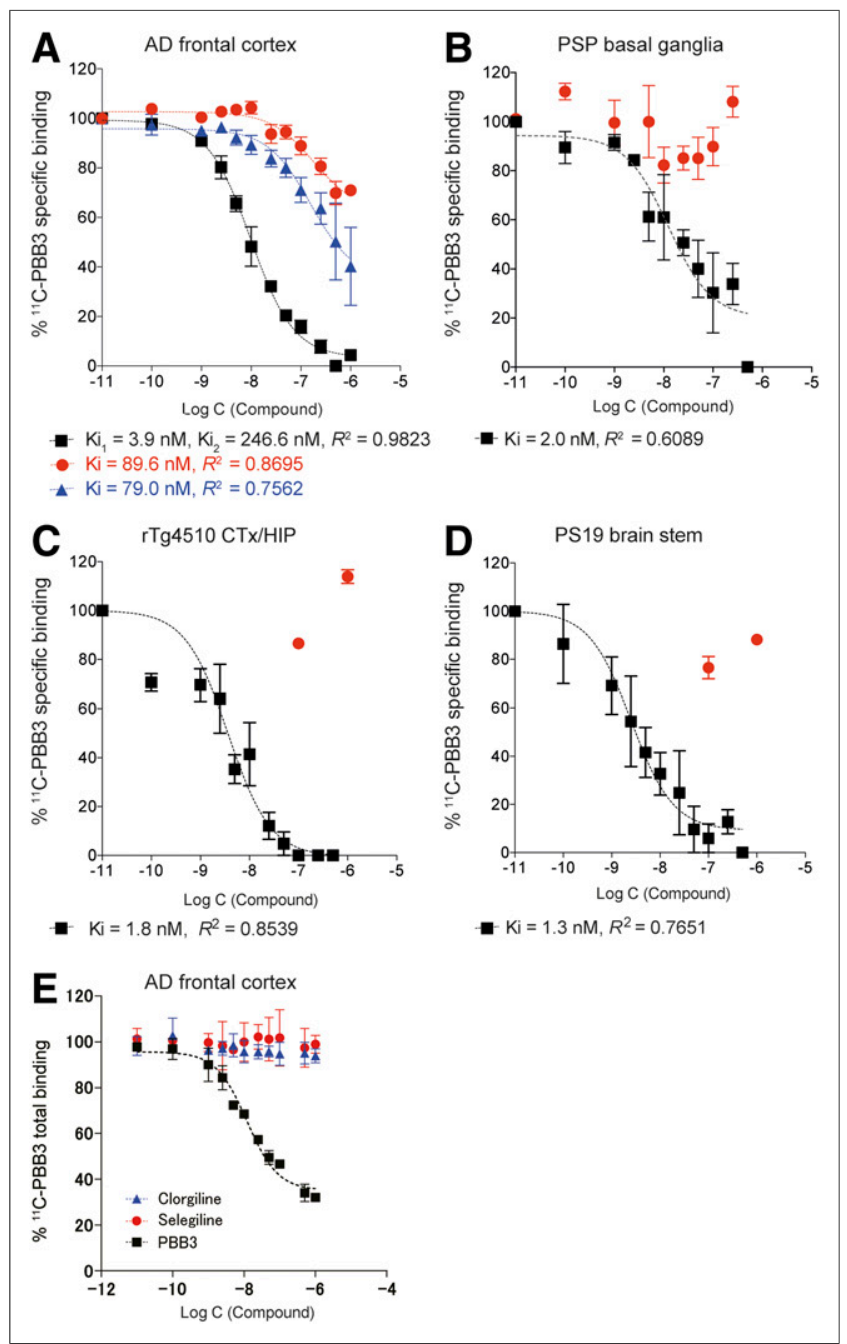

FIGURE 3. Assays of ${ }^{11} \mathrm{C}$-PBB3 binding in brain tissues derived from mouse models and $A D$ patient. Graphs show specific binding of ${ }^{11} \mathrm{C}$ PBB3 $(5 \mathrm{nM})$ in frontal cortex obtained from AD patient (A), basal ganglia from PSP patient (B), neocortex (CTx)/hippocampus (HIP) from rTg4510 mice (C, samples from 5 mice undergoing PET), and brain stem/spinal cord from PS19 mice (D, samples from three 13-mo-old mice). ${ }^{11} \mathrm{C}-\mathrm{PBB} 3$ binding was blocked in homologous fashion by nonlabeled PBB3 (black symbols) and in heterologous fashion by nonlabeled AV-1451 (red symbols) and BTA-1 (blue symbols). (E) No notable displacement of ${ }^{11} \mathrm{C}-\mathrm{PBB} 3$ binding by clorgyline or selegiline was observed in frontal cortex of $A D$, in contrast to significant blockade of radioligand binding by unlabeled PBB3. Data were generated from more than 3 independent experiments. binding was only minimally inhibited by AV-1451 at high concentrations (Figs. 3C and 3D).

The lack of competition between ${ }^{11} \mathrm{C}-\mathrm{PBB} 3$ and AV-1451 binding in PSP and transgenic mouse brain samples supports the view that ${ }^{11} \mathrm{C}$-PBB3 does not react with probable off-target binding components for $\mathrm{AV}-1451$, including monoamine oxidase A and ironcontaining deposits (24). Finally, minimal displacement of ${ }^{11} \mathrm{C}-\mathrm{PBB} 3$ binding was observed in the presence of the monoamine oxidase $\mathrm{A}$ inhibitor clorgyline or the monoamine oxidase B inhibitor selegiline in homogenates from the $\mathrm{AD}$ frontal cortex even at high concentrations (Fig. 3E), suggesting that ${ }^{11} \mathrm{C}-\mathrm{PBB} 3$ can barely react with off-target binding sites on monoamine oxidases.

\section{DISCUSSION}

The current findings provide in vitro and in vivo evidence for the conformational homology between pathologic tau assemblies in human tauopathies and mouse models as probed by ${ }^{11} \mathrm{C}-\mathrm{PBB} 3$. Binding assays of ${ }^{11} \mathrm{C}-\mathrm{PBB} 3$ in comparison with $\mathrm{AV}-1451$ have demonstrated that tau fibrils in transgenic mice overexpressing a single 4-repeat tau isoform particularly resemble straight filaments in 4-repeat tau disorders represented by PSP. These indications support the notion that PET imaging of the model animals along with postmortem investigations with ${ }^{11} \mathrm{C}$-PBB3 offers assay systems seamlessly linking nonclinical and clinical evaluations of candidates for therapeutic and diagnostic agents targeting the tau depositions. To further justify the use of the nonclinical ${ }^{11} \mathrm{C}$ PBB3 PET system with rTg4510 and PS19 mice, a more extensive in vitro analysis of the relevance between radioligand binding profiles in transgenic mouse and human tissues will be required with a larger sample size and different brain areas. There might be regional and interindividual differences in the conformational properties and neurotoxicity of pathologic tau assemblies within a single disease, although the present data using the AD frontal cortex and PSP basal ganglia (Figs. 3C and 3D) agreed with previous findings in different tangle-rich brain regions of patients with these illnesses (13).

As illustrated in Figure 3, ${ }^{11} \mathrm{C}-\mathrm{PBB} 3$ is capable of binding to tau deposits in the PSP and transgenic mouse brain tissues with a high affinity, and this binding was not blocked by AV-1451 even at a high concentration above $100 \mathrm{nM}$. This observation demonstrates the resemblance of straight filaments composed of 4-repeat taus in PSP and transgenic models, raising the possibility that PET scans of these mice will facilitate the development of a probe for highcontrast imaging of tau lesions in PSP and allied 4-repeat tauopathies. Such new imaging agents would be generated on the basis of the PBB3 backbone structure, in light of the current data.

In the $\mathrm{AD}$ brain, $\mathrm{A} \beta$ and tau aggregates share a common $\beta$-pleated sheet structure that is in principle reactive with diverse $\beta$-sheet ligands, but our present and previous results $(7,13)$ suggest that ${ }^{11} \mathrm{C}-\mathrm{PBB} 3$ at a concentration below $10 \mathrm{nM}$, which is comparable to the radioligand concentration in the brain achievable in a clinical PET study, primarily reacts with tau versus $A \beta$ deposits in the brains of $\mathrm{AD}$ patients. Indeed, the peak concentration of ${ }^{11} \mathrm{C}$ PBB3 is conceived to be $10 \mathrm{nM}$ or below in human and mouse PET studies, according to the current and previous (7) data, and is therefore considered to be close to the concentration of ${ }^{11} \mathrm{C}$-PBB3 (i.e., $5 \mathrm{nM}$ ) used for the current homogenate binding assays. Because BTA- 1 binds to A $\beta$ aggregates with high affinity, the blockade of specific ${ }^{11} \mathrm{C}-\mathrm{PBB} 3$ binding in $\mathrm{AD}$ tissues by $1-50$ nM BTA-1 should reflect the binding of ${ }^{11} \mathrm{C}-\mathrm{PBB} 3$ to $\mathrm{A} \beta$ deposits, 
which was found to be minimal (Fig. 3A). Meanwhile, the heterologous blockade by BTA-1 at high concentrations above $100 \mathrm{nM}$ is likely to stem primarily from the low-affinity binding of BTA-1 to tau fibrils. This finding agrees with previous autoradiographic binding measurements indicating that ${ }^{11} \mathrm{C}-\mathrm{PBB} 3$ at a concentration below $10 \mathrm{nM}$ reacts with mostly high-affinity components on tau fibrils, relative to low-affinity elements on $\mathrm{A} \beta$ fibrils, in $\mathrm{AD}$ brain sections. Moreover, the affinities of ${ }^{11} \mathrm{C}-\mathrm{PBB} 3$ for tau in the brains of $\mathrm{AD}$ patient and transgenic mouse were close to each other, validating the use of these animals for nonclinical characterization of ${ }^{11} \mathrm{C}-\mathrm{PBB} 3$ and in vivo screening of $\mathrm{PBB}$ derivatives for imaging $\mathrm{AD}$-type tau pathologies. Notwithstanding this supportive evidence, the properties of ${ }^{11} \mathrm{C}-\mathrm{PBB} 3$ and resemblance between $\mathrm{AD}$ and transgenic mouse tau fibrils will need to be further examined by increasing the sample size of $\mathrm{AD}$ tissues.

It is noteworthy that ${ }^{11} \mathrm{C}-\mathrm{PBB} 3$ binding in $\mathrm{AD}$ brain homogenates was only partially blocked by $\mathrm{AV}-1451$, with a $\mathrm{K}_{\mathrm{i}}$ value of $89.6 \mathrm{nM}$ (Fig. 3A), which is consistent with our previous report (13). Because specific binding of $5 \mathrm{nM}{ }^{11} \mathrm{C}-\mathrm{PBB} 3$ was barely inhibited by 1-50 nM AV-1451, PBB3 and AV-1451 may not share high-affinity binding components in $\mathrm{AD}$ tau filaments. In addition, the high-affinity binding of $5 \mathrm{nM}{ }^{11} \mathrm{C}-\mathrm{PBB} 3$ was only modestly blocked by AV-1451 at high concentrations (Fig. 3A). Hence, ${ }^{11} \mathrm{C}-\mathrm{PBB} 3$ and ${ }^{18} \mathrm{~F}-\mathrm{AV}-1451$ in living $\mathrm{AD}$ patients may detect different tau species that differentially contribute to neurotoxicity as implied by a previous in vitro assessment (13), and this probability will be examined by a head-to-head comparison of these 2 radioligands in the same human subjects.

The present work also indicates an intimate correlation between in vivo and in vitro binding of ${ }^{11} \mathrm{C}-\mathrm{PBB} 3$ in the same $\mathrm{rTg} 4510$ mice at 7-10 mo old. We defined PET VOIs in a subportion of the neocortex to circumvent technical issues of radioactivity spill-in and partial-volume effects stemming from the small volumes of the mouse brain regions, enabling quantification of the radioligand binding reflecting the number of binding components. Because we observed a marked colocalization of PBB3 fluorescence labeling and AT8 immunostaining in the brain sections derived from these mice, in vivo ${ }^{11} \mathrm{C}$-PBB3 $\mathrm{PET}$ is presumed to be able to robustly quantify insoluble tau aggregates constituted of phosphorylated tau molecules. Meanwhile, there still remains a possibility of inaccurate measurements of the radioligand binding caused by insufficient volumes of target VOIs, particularly in mice with severe pathologies, highlighting the need for further technical considerations in small-animal PET imaging.

According to the correlation between local brain atrophy and in vivo ${ }^{11} \mathrm{C}$-PBB3 binding in $\mathrm{rTg} 4510$ mice, neurons in the brains of these animals may die mainly at a pathologic stage characterized by the accumulation of PBB3-labeled inclusions, and our previous data also demonstrate that neuronal loss is less prominent in the absence of PBB3 positivity notwithstanding the high abundance of tau oligomers (25), suggesting a potential role of PBB3-positive inclusions in the advancement of the neurotoxicity. In the meantime, extracellular tau deposits dubbed ghost tangles were not found in our histochemical and immunohistochemical analyses of the brain sections collected from rTg4510 mice. This implies death of tanglebearing neurons followed by immediate elimination of tau aggregates or loss of tangle-negative neurons due to neurotoxicity of relatively immature tau assemblies. To examine these possibilities, longitudinal PET and intravital microscopic imaging experiments need to be conducted and are currently under way. In PET scans of patients with AD or mild cognitive impairment $(7,26)$, an increase in ${ }^{11} \mathrm{C}-\mathrm{PBB} 3$ binding has been reported to be correlated with either local atrophy or cognitive performance, suggesting that the neuronal loss in these diseases is mediated by molecular mechanisms linked to PBB3-positive tau fibrils similar to those in $\mathrm{rTg} 4510$ mice.

In contrast to the pathologies in rTg4510 mice, AT8-immunoreactive tau deposits in the neocortex/hippocampus of PS19 mice barely reacted with PBB3. Meanwhile, a pronounced increase of tau lesions doubly positive for AT8 and PBB3 occurred in the brain stem and spinal cord of the PS19 strain. Because regional atrophy was severe in the neocortex/hippocampus and modest in the brain stem and spinal cord of these animals $(7,21)$, the concurrence of PBB3-negative, AT8-positive inclusions with loss of neurons and absence of ghost tangles in the hippocampus of PS19 mice might indicate the neurotoxicity of PBB3-negative tau assemblies, or immediate elimination of neurons bearing PBB3positive tau tangles. Furthermore, the PBB3 negativity in the PS19 hippocampus may be attributable to the formation of regionspecific conformers and posttranslational modifications of tau molecules, leading to the loss of their reactivity with PBB3. The reactivity of different PET probes with tauopathy mouse models in addition to rTg4510 and PS19 mice is also of significant interest. A previous PET study documented that another tau PET ligand, ${ }^{18} \mathrm{~F}-\mathrm{THK} 5117$, enabled quantification of tau deposits in 2 different tau transgenic mouse models (27), whereas the relationships between tau PET signals and regional atrophy in these strains remain undetermined. Furthermore, it should be noted that THK5117 and related quinoline derivatives may cross-react with monoamine oxidase B (28), which is abundantly present in activated astrocytes, whereas ${ }^{18} \mathrm{~F}-\mathrm{AV}-1451$ may bind to monoamine oxidase A (24). Our in vitro assays indicate that ${ }^{11} \mathrm{C}-\mathrm{PBB} 3$ binding in $\mathrm{AD}$ brain homogenates does not compete with inhibitors of either monoamine oxidase $\mathrm{A}$ or monoamine oxidase $\mathrm{B}$, and therefore ${ }^{11} \mathrm{C}$-PBB3-PET signals in human and mouse brains are considered to reflect tau lesions, not other neuropathologic events such as astrogliosis enriched with monoamine oxidase B.

\section{CONCLUSION}

Our PET and MRI analyses of tau transgenic mice in conjunction with postmortem radiochemical, histochemical, and immunohistochemical assays have proven the applicability of ${ }^{11} \mathrm{C}-\mathrm{PBB} 3$ to the research on neurodegenerative tauopathies reciprocally linking nonclinical and clinical evidence. The findings obtained here have also revealed the distinct utility of rTg4510 and PS19 models for assessing putative etiologic pathways triggered by either PBB3-positive or PBB3-negative tau multimers.

\section{DISCLOSURE}

This work was supported by grants-in-aid for Brain Mapping by Integrated Neurotechnologies for Disease Studies (Brain/MINDS; 15653129) to Tetsuya Suhara and Makoto Higuchi; Research and Development Grants for Dementia (16768966) to Makoto Higuchi and the COI STREAM award for MRI devices to Ichio Aoki from the Japan Agency for Medical Research and Development; 2 grants-in-aid for Scientific Research to Bin Ji (15K09979) and Makoto Higuchi (16678815) and a postdoctoral Fellowship to Ruiqing Ni (PE15760) from the Japan Society for the Promotion of Science. No other potential conflict of interest relevant to this article was reported. 


\section{ACKNOWLEDGMENTS}

We thank Prof. John Q. Trojanowski and Prof. Virginia M.-Y. Lee (Center for Neurodegenerative Disease Research, University of Pennsylvania) for kindly providing human brain tissues and PS19 mice; Prof. Jada Lewis of the University of Florida for supporting analyses of rTg4510 mice; and Takeharu Minamihisamatsu, Shoko Uchida, Sayuri Sasaki, Sayaka Shibata, Nobuhiro Nitta, and Yoshikazu Ozawa for technical assistance.

\section{REFERENCES}

1. Lee VM, Goedert M, Trojanowski JQ. Neurodegenerative tauopathies. Annu Rev Neurosci. 2001;24:1121-1159.

2. Higuchi M, Lee VM, Trojanowski JQ. Tau and axonopathy in neurodegenerative disorders. Neuromolecular Med. 2002;2:131-150.

3. Murray ME, Graff-Radford NR, Ross OA, Petersen RC, Duara R, Dickson DW. Neuropathologically defined subtypes of Alzheimer's disease with distinct clinical characteristics: a retrospective study. Lancet Neurol. 2011;10:785-796.

4. Nelson PT, Alafuzoff I, Bigio EH, et al. Correlation of Alzheimer disease neuropathologic changes with cognitive status: a review of the literature. J Neuropathol Exp Neurol. 2012;71:362-381.

5. Khanna MR, Kovalevich J, Lee VM, Trojanowski JQ, Brunden KR. Therapeutic strategies for the treatment of tauopathies: hopes and challenges. Alzheimers Dement. 2016;12:1051-1065.

6. Villemagne VL, Fodero-Tavoletti MT, Masters CL, Rowe CC. Tau imaging: early progress and future directions. Lancet Neurol. 2015;14:114-124.

7. Maruyama M, Shimada H, Suhara T, et al. Imaging of tau pathology in a tauopathy mouse model and in Alzheimer patients compared to normal controls. Neuron. 2013;79:1094-1108.

8. Okamura N, Furumoto S, Harada R, et al. Novel ${ }^{18} \mathrm{~F}-$ labeled arylquinoline derivatives for noninvasive imaging of tau pathology in Alzheimer disease. $\mathrm{J} \mathrm{Nucl}$ Med. 2013;54:1420-1427.

9. Harada R, Okamura N, Furumoto S, et al. ${ }^{18}$ F-THK5351: a novel PET radiotracer for imaging neurofibrillary pathology in Alzheimer disease. J Nucl Med. 2016; 57:208-214.

10. Chien DT, Bahri S, Szardenings AK, et al. Early clinical PET imaging results with the novel PHF-tau radioligand [F-18]-T807. J Alzheimers Dis. 2013;34:457-468.

11. Chien DT, Szardenings AK, Bahri S, et al. Early clinical PET imaging results with the novel PHF-tau radioligand [F18]-T808. J Alzheimers Dis. 2014;38:171-184.

12. Johnson KA, Schultz A, Betensky RA, et al. Tau positron emission tomographic imaging in aging and early Alzheimer disease. Ann Neurol. 2016;79:110-119.
13. Ono M, Sahara N, Kumata K, et al. Distinct binding of PET ligands PBB3 and AV1451 to tau fibril strains in neurodegenerative tauopathies. Brain. 2017;140:764-780.

14. Marquié M, Normandin MD, Meltzer AC, et al. Pathological correlations of [F-18]AV-1451 imaging in non-Alzheimer tauopathies. Ann Neurol. 2017;81:117-128.

15. Hammes J, Bischof GN, Giehl K, et al. Elevated in vivo $\left[{ }^{18} \mathrm{~F}\right]-\mathrm{AV}-1451$ uptake in a patient with progressive supranuclear palsy. Mov Disord. 2017;32:170-171.

16. Coakeley S, Cho SS, Koshimori Y, et al. Positron emission tomography imaging of tau pathology in progressive supranuclear palsy. J Cereb Blood Flow Metab. 2017:37:3150--3160.

17. Ishiki A, Harada R, Okamura $\mathrm{N}$, et al. Tau imaging with $\left[{ }^{18} \mathrm{~F}\right] \mathrm{THK}-5351$ in progressive supranuclear palsy. Eur J Neurol. 2017;24:130-136.

18. Bibow S, Mukrasch MD, Chinnathambi S, et al. The dynamic structure of filamentous tau. Angew Chem Int Ed Engl. 2011;50:11520-11524.

19. Murray ME, Kouri N, Lin WL, Jack CR Jr, Dickson DW, Vemuri P. Clinicopathologic assessment and imaging of tauopathies in neurodegenerative dementias. Alzheimers Res Ther. 2014;6:1.

20. Santacruz K, Lewis J, Spires T, et al. Tau suppression in a neurodegenerative mouse model improves memory function. Science. 2005;309:476-481.

21. Yoshiyama Y, Higuchi M, Zhang B, et al. Synapse loss and microglial activation precede tangles in a P301S tauopathy mouse model. Neuron. 2007;53:337-351.

22. Ji B, Maeda J, Sawada M, et al. Imaging of peripheral benzodiazepine receptor expression as biomarkers of detrimental versus beneficial glial responses in mouse models of Alzheimer's and other CNS pathologies. J Neurosci. 2008; 28:12255-12267.

23. Maeda J, Zhang MR, Okauchi $\mathrm{T}$, et al. In vivo positron emission tomographic imaging of glial responses to amyloid- $\beta$ and tau pathologies in mouse models of Alzheimer's disease and related disorders. J Neurosci. 2011;31:4720-4730.

24. Marquié M, Normandin MD, Vanderburg CR, et al. Validating novel tau positron emission tomography tracer [F-18]-AV-1451 (T807) on postmortem brain tissue. Ann Neurol. 2015;78:787-800.

25. Sahara N, Ren Y, Ward S, Binder LI, Suhara T, Higuchi M. Tau oligomers as potential targets for early diagnosis of tauopathy. J Alzheimers Dis. 2014;40 (suppl 1):S91-S96.

26. Shimada H, Kitamura $S$, Shinotoh $H$, et al. Association between $A \beta$ and tau accumulations and their influence on clinical features in aging and Alzheimer's disease spectrum brains: A $\left[{ }^{11} \mathrm{C}\right] \mathrm{PBB} 3-\mathrm{PET}$ study. Alzheimers Dement (Amst). 2016;6:11-20

27. Brendel M, Jaworska A, Probst F, et al. Small-animal PET imaging of tau pathology with ${ }^{18} \mathrm{~F}-\mathrm{THK} 5117$ in 2 transgenic mouse models. J Nucl Med. 2016;57:792-798.

28. Ng KP, Pascoal TA, Mathotaarachchi S, et al. Monoamine oxidase B inhibitor, selegiline, reduces ${ }^{18} \mathrm{~F}$-THK5351 uptake in the human brain. Alzheimers Res Ther. 2017;9:25. 